Aus der chirurgischen Klinik in Lyon (Direktor: Prof. Dr. A. Poncet).

\title{
Über einige neue Indikationen der Durchschneidung der hinteren Wurzeln.
}

Von Privatdozent Dr. René Leriche.

(Mit 2 Abbildungen.)

Die Durchschneidung der hinteren Wurzeln ist bis jetzt nur in zwei Fällen indiciert gewesen : einmal wurde sie ausgeführt, um die unerträglichen gastrischen Krisen zu lindern oder um die hochgradige zentripetale Reizung herabzusetzen, welche spastische Erscheinungen oder Tumor unterhält.

Nach den Erfahrungen, über die man heute ${ }^{1}$ ) über diese schwierige, aber keineswegs tödliche Operation, wie man oft behauptet hat, verfügt, kann man ohne Bedenken den Kreis ihrer Indikationen zu erweitern suchen. Von diesem Gedankengang ausgehend, erweist sich ihre Anwendung als zweckmäßig, wenn es gilt, eine irgendwie beschaffene trophische Störung zu behandeln. Ich will damit sagen, daB es zweifellos von Nutzen wäre, Durchschneidungen von hinteren Wurzeln bei hartnäckigen peripheren Läsionen vorzunehmen, die sich bei näherer Betrachtung als Erscheinungen von seiten der Wurzeln und Nerven ergeben.

Es existieren verschiedene Krankheitszustände, die man zurzeit als solche ansehen kann:

I. Zunächst der Herpes intercostalis. Es dürfte sich erübrigen, näher auszuführen, daß dieser abgesehen von Ausnahme-

I) Ich habe 124 Fälle von Försterscher Operation wegen spastischer Paraplegie, 62 Fälle wegen gastrischer Krisen, 21 wegen verschiedener Neuralgien zusammenstellen können. Ich selbst habe bis September $19127 \mathrm{mal}$ die Förstersche Operation ausgeführt, nämlich $3 \mathrm{mal}$ bei Tabikern, $3 \mathrm{mal}$ wegen Paralysis agitans und I mal wegen Herpes. 
fällen nicht das Produkt einer interkostalen Neuritis ist. Die klassischen Autoren zitieren nach Belieben die Liste der zeitgenössischen Autoren, welche die schon im Jahre $186 \mathrm{I}$ von $v$. Baerensprung, dann von $\mathrm{Cha}$ r cot beschriebenen Läsionen an den Nervenknoten wieder ausgegraben haben. Andererseits ge-

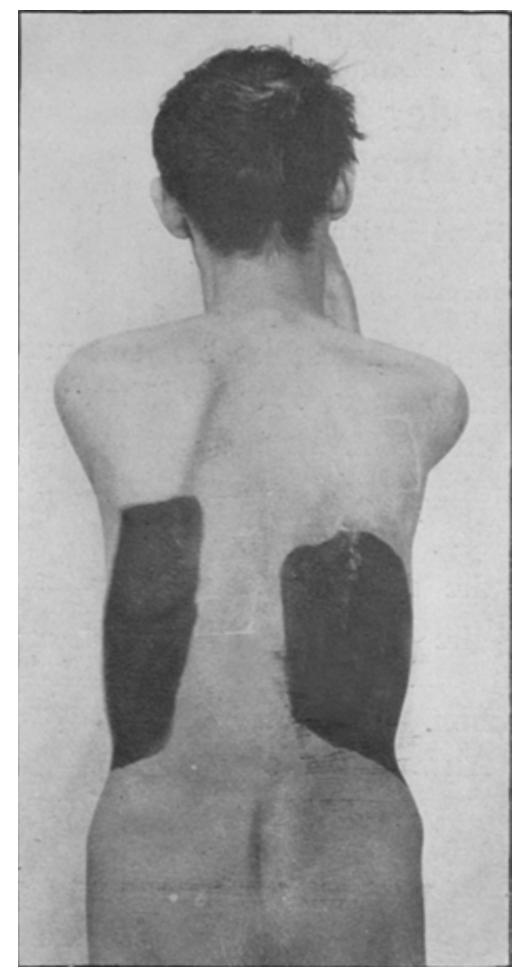

Fig. I. Die Photographie zeigt die hypästhetische Zone, die nach Extraktionen des 7., 8., 9., 1 o. u. 1 I. Interkostalnerven beobachtet wurde. Nach hinten zu besteht eine Zone von normaler Sensibilität infolge d. Erhaltung der hinteren Scite des Dorsalnerven. nügt in vielen Fällen die klinische Untersuchung allein, um die Wurzeltopographie der Zostereruption zu ermitteln. Von diesem Standpunkt aus scheint mir eine einfache Feststellung einen peremptorischen Beweis zu liefern, der weit hergeholt erscheint: in der Regel erreichen die Herpesbläschen die Linie der Dornfortsätze und bedecken auf diese Weise nach hinten eine Region, in welcher der Nervus intercostalis nicht verläuft. Die beiden nachstehenden Photographien zeigen dies deutlich.

Hinge die Eruption von einer Stammneuritis ab, so müBte sie diese Zone respekticren, die von dem hinteren Ast des gemischten Nerven versorgt wird. Sie tut es nicht, folglich ist der Herpes eine Entzündung der Nervenwurzel.

Während es nun vorübergehende Entzündungen der Nervenwurzeln und Herpesarten mit raschem Verlauf gibt, gibt es andere sehr hartnäckige, die stets zu Rezidiven führen und äußerst schmerzhaft sind. Die Durchschneidung der kranken Wurzeln müßıc logischerweise die Therapie $\left.\alpha \alpha \tau^{\prime} \dot{k} \xi \circ \chi \dot{\eta}\right)$ dieser Fälle sein, Ich glaubc nicht, daß man je daran gedacht hat. Ich habe ganz 
Uber einige neue Indikationen der Durchschneidung der hinteren Wurzeln. 487

kürzlich in einem derartigen He:pesfall die 4. und 5. Dorsalwurzel der entsprechenden Seite durchschnitten. Der Thorax war wie infiltriert. Die Berührung löste an jener Stelle heftige Schmerzen aus. Außerdem bestanden daselbst irreparable Narben, ein großes Bläschen mit sehr empfindlichem rötlichen Vorhof. Außerdem belästigten lanzinierende Paroxysmen die Patientin andauernd.

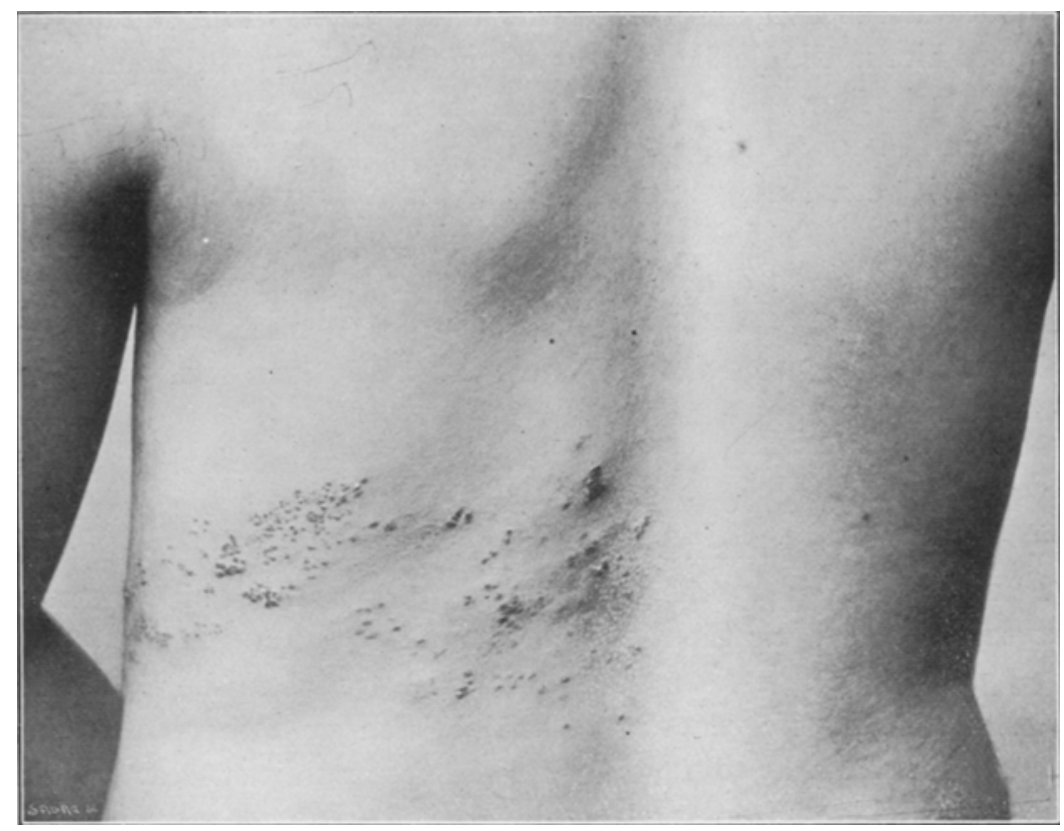

Fig. 2. Herpes thoracicus, bei einem 32 jährigen Manne nach einer sehr heftigen hyperchlorhydrischen Krise entstanden.

48 Stunden nach der Operation war das Bläschen eingeschrumpft, ausgetrocknet und buchstäblich vernarbt.

Die Mamma erwies sich bei der Palpation als unempfindlich und glich vollständig wieder der anderen. Das therapeutische Resultat war mit überraschender Schnelligkeit erzielt worden. Die Einzelheiten über diesen Fall sollen demnächst publiziert werden. Für den Augenblick genügt es mir, die nützliche Wirkung der Nervendurchschneidung auf die gestörte Trophik einer Wurzel gezeigt zu haben. 
2. Eine häufigere Indikation dieser trophischen Wurzeldurchschneidung würde vielleicht das Mal perforant der Planta pedis liefern.

Es handelt sich nicht darum, die Wurzeldurchschneidung bei allen trophischen Ulzerationen des Fußes auszuführen. Bevor man daran geht, soll man es mit einer Ruhekur und aseptischer Behandlung versuchen, soll alle Register der peripheren Nervenoperationen ziehen, die Neurorexis und speziell die Dehnung des Sympathicus, die Jaboula y unter dem Namen „dénudation“ der Arteria femoralis beschrieben hat. Ich habe für meinen Teil zwei Fälle beobachtet, wo die Jaboula y sche Methode zu einer schnellen Vernarbung eines hartnäckigen Plantargeschwürs führte. Aber es gibt Fälle, in denen all die genannten Methoden versagen. Es gibt andere, in denen das $\mathrm{Mal}$ perforant beim ersten Gang rezidiviert. Man muß in solchen Fällen den Gedanken einer peripheren Neuritis fallen lassen, einen radikulären Ursprung annehmen und demgemäß verfahren.

Es ist sogar wahrscheinlich, daB man manchmal durch die bloße klinische Untersuchung das radikuläre Mal perforant diagnostiziert. In solchen Fällen würde die Durchschneidung der 5. Lumbal- und der I. Sakralwurzel wahrscheinlich eine prompte Besserung der Ulzeration zur Folge haben.

3. Es gibt eine letzte Krankheit, die, trotzdem die Sache auf den ersten Blick eigenartig erscheint, sich vom therapeutischen Standpunkt aus an die vorhergehenden anschließt. Es ist dies die hartnäckige $\mathrm{Hyperchlorhydrie.} \mathrm{Was} \mathrm{man} \mathrm{auch} \mathrm{sagen}$ mag, es existieren zweifellos Zustände von schmerzhafter, gastrischer und intestinaler Hypersekretion, abgesehen von den Magen- und Duodenalgeschwüren. Es gibt Patienten, die an furchtbaren Krisen leiden, Hypersekretion haben, die keinerlei klinische oder röntgenologische Symptome eines Ulcus oder einer Stenose zeigen, bei denen ein lokaler Eingriff keinen Befund ergibt und sich als gänzlich zwecklos herausstellt. Nach Ansicht einiger Autoren soll es sich um eine unausgebildete Tabes mit gastrischem Beginn handeln. Wie es sich auch mit der Pathogenese dieser Zustände verhalte, Tatsache ist, daß solche Patienten existieren. Sie sind gering an Zahl, was aber kein Grund ist, um sie zu vernachlässigen. Ihr Leiden be- 
thber einige neue Indikationen der Durchschneidung der hinteren Wurzeln. 489

steht hauptsächlich in ihrer Hypersekretion, wozu dann noch in zweiter Linie ihre Hyperästhesie kommt. Es erscheint nicht unberechtigt, daran zu denken, die lokale Behandlung zu verlassen und durch Beeinflussung des Nervensystems die Sekretionswerte und damit zugleich ihre sensiblen Beschwerden herabzusetzen.

Von den beiden Gruppen von Nerven, die zum Magen hinziehen, sind diejenigen, die vom Vagus abgehen, vor allem motorisch und versorgen nur die psychische Sekretion. Im Gegensatz dazu sind die Sympathicusäste vor allem sensibel und zu gleicher Zeit sekretorisch. Obgleich die Magensekretion auch nach ihrer vollständigen Durchschneidung fortbesteht, ist ihre Bedeutung doch sicher eine sehr große. Erscheint die Annahme nicht logisch, daß Hypersekretionen durch eine Neuritis des Sympathicus zustande kommen können, daß die beginnende Läsion entweder peripher oder im Gegensatz dazu radikulär ist? Diese Ansicht läßt sich gewiß hören. Ich für meinen Teil werde nicht zögern in einem solchen Fall, der durch einen kompetenten Kliniker genauestens untersucht ist, den Sympathicus entweder durch Dehnung des Plexus solaris anzugreifen (was den Vorteil einer direkten Besichtigung des Magens und der Nachbarorgane mit sich bringen würde) oder im Fall eines Mißerfolges eine Durchschneidung der hinteren Dorsalwurzeln, und zwar der 5., 6., 7., 8., 9. und Io. Dorsalwurzel vorzunehmen.

Ein ähnlicher Gedankengang läßt das Prinzip eines gleichartigen Eingriffes in manchen Fällen von hartnäckiger Colitis muco-membranacea berechtigt erscheinen, die in sich das charakteristische Merkmal der Neuritis des Bauchsympathicus tragen und die nichts im Abdomen selbst hinreichend erklärt.

Ich beschränke mich darauf, das Problem hiermit zur Diskussion zu stellen, das in dem eng begrenzten Rahmen der Ausnahmefälle, für die ich es umschreibe, einer weiteren Untersuchung gewiß wert ist. 